\title{
Patrones de desplazamiento de peces nativos en el Río San Pedro (cuenca del Río Valdivia, Chile)
}

\author{
Movement patterns of the native fish fauna of the San Pedro River (Valdivia River Basin, \\ Chile)
}

\author{
Priscila Piedra ${ }^{1 *}$, Evelyn Habit ${ }^{1}$, Alejandra Oyanedel ${ }^{1}$, Nicole Colin ${ }^{1}$, Katherin Solis-Lufí ${ }^{1}$, Jorge \\ González ${ }^{1}$, Alfonso Jara ${ }^{1}$, Néstor Ortiz ${ }^{1}$ \& Roberto Cifuentes ${ }^{1}$ \\ ${ }^{1}$ Unidad de Sistemas Acuáticos. Centro de Ciencias Ambientales Eula-Chile. Universidad de Concepción. Casilla 160-C. \\ Concepción. \\ *E-mail: ppiedra@udec.cl
}

\begin{abstract}
RESUMEN
Los peces nativos chilenos se caracterizan por ser de tamaño pequeño $(<30 \mathrm{~cm})$. Por ello, generalmente se les menciona como de baja capacidad de natación y desplazamiento. Sin embargo, no existen estudios empíricos que lo demuestren. En este estudio describimos los patrones de movimiento de seis especies nativas en el río San Pedro (Aplochiton taeniatus, Galaxias maculatus, Galaxias platei, Diplomystes camposensis, Basilichthys australis, Percilia gillissi y Percichthys trucha), utilizando técnicas de marcaje-recaptura mediante pinturas elastoméricas y Pit-tags. Nuestros resultados sugieren que la mayor parte de las especies presentan desplazamientos pasivos, recorriendo distancias de hasta aproximadamente 40 $\mathrm{km}$ en el sentido de la corriente. También fueron frecuentes los movimientos entre riberas, aún en zonas de alta turbulencia en el río. No se evidenciaron claros movimientos activos hacia aguas arriba, pero el análisis de los resultados en conjunto con información de la estructura genética, abundancias y distribución de las especies en la cuenca, nos permite concluir que los patrones de desplazamiento de todas las especies estudiadas son relevantes para la mantención de su dinámica metapoblacional.
\end{abstract}

Palabras clave: Peces nativos, desplazamiento, marcaje-recaptura, Pit-tags, Chile.

\begin{abstract}
The Chilean native fish are characterized for having small sizes $(<30 \mathrm{~cm})$. Due to this, they are generally described with a low swimming capacity and movement; nevertheless, there is no empirical study to show it. In this study we describe the movement patterns of six native species in the San Pedro River (Aplochiton taeniatus, Galaxias maculatus, Galaxias platei, Diplomystes camposensis, Basilichthys australis y Percilia gillissi) by using marking-recapture techniques with elastomeric inks and Pit tags. Our results suggest that most of the species show passive movements, crossing distances up to about $40 \mathrm{~km}$ in the flow direction. Movements between river banks were also frequent, even in zones of high turbulence of the river. Active movements upstream were note clear, but the analysis of the results altogether with information of the genetic structure, abundances and distribution of the species in the watershed, allows us to conclude that the movement patterns of all species studied are relevant for the maintenance of their metapopulation dynamics.
\end{abstract}

KeYwords: native fish, movement, mark-recapture, Pit-tags, Chile.

\section{INTRODUCCIÓN}

Los desplazamientos de la fauna íctica de sistemas fluviales, están asociados principalmente a estrategias reproductivas y alimentarias que les permiten acceder a los recursos necesarios para su sobrevivencia (Eiler 2000). Por ello, el movimiento es un factor fundamental en la biología y ecología de los peces (Schlosser 1991; Gowan et al. 1994; Rodriguez 2002; Allen et al. 2010). En la amplia gama de movimientos realizados por la fauna íctica, se encuentran aquellos de gran escala efectuados por peces diádromos que se desplazan entre el agua dulce y el mar (McDowall 2002), y otros de carácter más local. Estos últimos son menos extensivos y no sincrónicos (Albanesse et al. 2004), pero son igualmente 
de gran significado ecológico. Tales movimientos locales permiten colonizar nuevos hábitats (Taylor 1997; Lonzarich et al. 1998), o moverse entre microhábitats, generando una alta dinámica espacial y temporal de las poblaciones en un sistema fluvial (Schlosser \& Angermeier 1995). Estos movimientos pueden estar gatillados por factores endógenos (e.g. reproducción o alimentación) o exógenos, como perturbaciones naturales (inundaciones, sequías, erupciones volcánicas) o antropogénicas.

Dadas las características hidrológicas de los ríos, los desplazamientos de los peces en ellos pueden ser pasivos o activos. Los primeros corresponden a la deriva de organismos originados por los incrementos de caudal durante las épocas de crecida, los que generan un desplazamiento en el sentido de la corriente, unidireccional. En tanto, los movimientos activos son aquellos relacionados con el comportamiento. Estos pueden asociarse con los ciclos diarios de luz, búsqueda de alimento o refugio. Tanto los movimientos pasivos como los activos aumentan la probabilidad de sobrevivencia y por ende la probabilidad de asegurar los eventos reproductivos (Lucas \& Baras 2000).

Entre los factores que influyen en el tipo y magnitud de los desplazamientos que efectúan los peces de agua dulce están el tamaño corporal, factor de condición y tasa de crecimiento (Beamish 1978, Albanese et al. 2004, Lauder 2005). Las especies nativas de sistemas continentales chilenos, se caracterizan por ser de pequeño tamaño $(<30 \mathrm{~cm}$ de longitud) (Vila et al. 1999), por lo que generalmente se les indica como de baja capacidad de movimiento. Sin embargo, no existen antecedentes empíricos del tipo y magnitud de los desplazamientos de peces nativos en Chile. En consideración a las especiales características hidrológicas de los ríos chilenos, (Niemeyer \& Cereceda 1984), se consideró de importancia describir los patrones de desplazamiento de ocho especies nativas presentes en el río San Pedro: Aplochiton taeniatus (Jenyns 1842), Aplochiton zebra (Jenyns 1842), Galaxias maculatus (Jenyns 1842), Galaxias platei (Steindachner 1898), Diplomystes camposensis (Arratia 1987), Basilichthys australis (Eigenmann 1927), Percilia gillissi (Girard 1855) y Percichthys trucha (Valenciennes 1833), mediante la aproximación de marcaje - recaptura, utilizando como marcas la inyección subcutánea de pinturas elastoméricas y Pit-Tags.

\section{MATERIALES Y METODOS}

Área de ESTUdio y Muestreo

El área de estudio correspondió al río San Pedro (39 46, - 39 51' S), tributario de la cuenca del río Valdivia (10244 $\mathrm{km}^{2}$ ). Esta cuenca la compone una cadena de ocho lagos de origen glaciar y características oligotróficas que nace desde Argentina, y está compuesta por los siguientes lagos: Lácar, Pirehueico, Neltume, Pellaifa, Calafquén, Pullinque, Panguipulliy Riñihue. Esteúltimo corresponde al que da origen al río San Pedro (Fig.1). Este sistema fluvial posee un régimen hidrológico pluvio-nival, con bajo número de tributarios. En él se identifican tres zonas hidrogeomorfológicas. La primera corresponde al desagüe del lago Riñihue e inicio del río San Pedro, zona de mayor profundidad (hasta $25 \mathrm{~m}$ ), con baja velocidad de corriente y sustratos arcillosos compactados. La segunda es un área de laderas abruptas, con mayor pendiente, donde el cauce presenta mayor velocidad de corriente, generando hábitats de rápidos junto a la presencia de riberas rocosas. Por último, la zona donde el río ingresa en el valle, con menor pendiente y con hábitats mixtos entre rápidos y pozas, principalmente someras. En este río, y abarcando las tres áreas hidrogeomorfológicas, se establecieron 35 sitios de muestreo, ubicados desde el lago Riñihue hasta la confluencia con el río Quinchilca (Tabla 1). En cada punto de muestreo se realizó la captura de individuos mediante pesca eléctrica (Smith \& Root modelo LR-24), abarcando en cada evento de pesca la totalidad de hábitats ribereños disponibles. El estudio se realizó entre octubre 2005 y marzo 2008, efectuando 465 días de muestreos en total, en los 35 sitios considerados.

Los individuos capturados fueron anestesiados utilizando BZ-20 en una solución razón $1 \mathrm{~mL}: 5 \mathrm{~L}$ de agua. Se registró el peso $(\mathrm{g})$ mediante balanza digital $(0,01 \mathrm{~g})$ y se midió longitud estándar (LS) con ictiómetro de $0,1 \mathrm{~cm}$ de precisión. En cada evento de pesca, se marcó la totalidad de los individuos mayores a $2,5 \mathrm{~cm}$ LS, ya que peces de menor talla presentaron alta labilidad. Además se verificó si alguno correspondía a una recaptura. La técnica de marcaje utilizada dependió del tamaño y peso de cada individuo. Luego del marcaje cada individuo fue puesto en un sistema de recuperación post-anestesia y posteriormente fue devuelto a su hábitat.

Marcaje con elastomer: El proceso de marcaje con elastomer se realizó in situ en cada muestreo. Este marcaje consistió en una inyección sub-epidérmica de pintura elastomérica. Con el fin de diferenciar la zona de origen de marcaje del pez, se realizaron marcas de distintos colores en cinco zonas corporales, definidas según la época del año y la zona del río (Tabla 2). Las zonas del cuerpo en las que se realizó el marcaje correspondieron a la base de las aletas dorsal, pectoral, pélvica, anal y caudal (Fig. 2), por ser más translúcidas. Con el fin de interpretar los movimientos efectuados por los peces marcados, se establecieron cuatro zonas de marcaje a lo largo del río (Fig.1b, Tabla 1), cada una de ellas fue identificada con distinto color según la época del año (Tabla 2). Además, para determinar la existencia de movimientos transversales en el río (entre riberas), las marcas fueron realizadas en el lado derecho o izquierdo del pez, según la ribera en las que fueran capturados. 
Con el fin de conocer los desplazamientos de cada especie se analizó el número de individuos recapturados y las distancias entre los sitios de origen de marcaje y sitios de recaptura. Además, se estimó el tiempo máximo y mínimo de residencia para los individuos recapturados durante el año 2007. El tiempo máximo corresponde al periodo entre el primer mes que se marcó un individuo hasta el momento de su última recaptura. El tiempo mínimo de residencia corresponde al periodo existente entre el último mes que se le realizó una marca a un individuo recapturado hasta su última recaptura.

Implante de Pit-Tags: El implante de Pit-tag (modelo FS 2001, Biomark), se realizó solo en peces mayores a 4,5 g de peso para asegurar su sobrevivencia y para evitar que el implante afecte la capacidad natatoria y comportamiento. Todos los peces de esas características fueron trasladados al laboratorio y mantenidos en acuarios previamente desinfectados con Duplalim ${ }^{\circledR}$ (Veterquímica) en solución
1:200, con recambio de agua constante durante 24 horas para su aclimatación. Cada pez fue anestesiado mediante solución de benzocaína anestésico-tranquilizante para peces $\left(\mathrm{BZ}-20^{\circledR}\right)$ en solución $1 \mathrm{~mL}: 5 \mathrm{~L}$ BZ-20 antes de la inyección de Pit-tag en la cavidad abdominal. Previamente se registraron sus datos básicos (peso y talla) y el número de código del pit-tag implantado. Luego los peces fueron mantenidos en recuperación y observación por alrededor de tres días para verificar la cicatrización de la herida y la retención del Pit- tag. Luego fueron devueltos al mismo sitio y hábitat donde fueron capturados. Una vez recapturado un individuo implantado con Pit-tag, se le identificó utilizando un lector modelo EX TM (Biomark) el cual entrega el código único de identificación. En base a la comparación de los datos obtenidos al momento de su primera captura y aquellos obtenidos durante su recaptura, se analizó su desplazamiento y la tasa de crecimiento (talla y peso) de cada individuo.

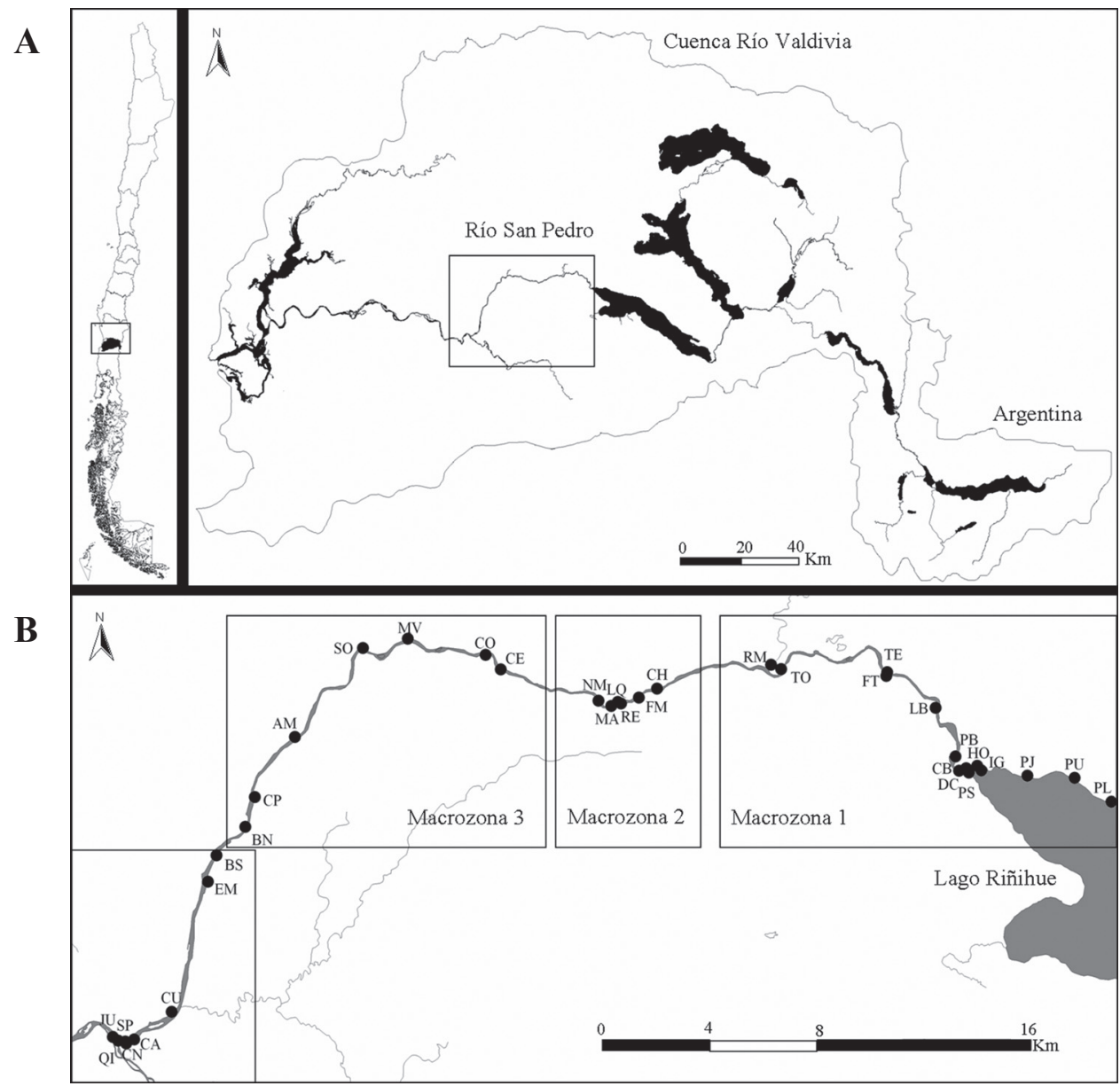

Figura 1. Ubicación de la zona de estudio. (a) Esquema de la cuenca del río Valdivia. (b) Río San Pedro. Los cuadrantes indican las cuatro zonas de marcaje $(1,2,3,4)$ y se muestran los códigos de los sitios de muestreo.

Figure 1. Study area localization. (a) Valdivia River Basin. (b) San Pedro River. Squares represent the four mark zones (1, 2, 3, 4) and the sampling site codes are shown. 
TABLA 1. Ubicación geográfica de los sitios de muestreo en zona de marcaje, ordenados desde el Lago Riñihue hacia aguas abajo.

TABLE 1. Sampling site and mark zones localizations, going from the Riñihue Lake downstream.

\begin{tabular}{|c|c|c|c|c|}
\hline \multirow[t]{3}{*}{ Zona Marcaje } & \multirow{3}{*}{ Nombre del Sitio } & \multirow{3}{*}{ Código } & \multirow{2}{*}{ UTM } & \multirow[t]{2}{*}{ UTM } \\
\hline & & & & \\
\hline & & & $\mathrm{X}$ & $\mathrm{Y}$ \\
\hline \multirow[t]{9}{*}{1} & Playa & PL & 722328 & 5593120 \\
\hline & Puntilla & PU & 721189 & 5593820 \\
\hline & Picá de Javier & PJ & 719743 & 5593884 \\
\hline & Isla Grande & IG & 718328 & 5594046 \\
\hline & Playa Sur & PS & 717940 & 5593975 \\
\hline & Cabañas & $\mathrm{CB}$ & 718198 & 5594178 \\
\hline & Hotel & $\mathrm{HO}$ & 717859 & 5594106 \\
\hline & Desembocadura Catalina & $\mathrm{DC}$ & 717642 & 5594034 \\
\hline & Piedra Blanca & PB & 717526 & 5594451 \\
\hline \multirow[t]{11}{*}{2} & Los Bajos & LB & 716917 & 5595892 \\
\hline & Carmen del Trafún Este & TE & 715426 & 5596941 \\
\hline & Fundo Tornagaleones & FT & 715400 & 5596832 \\
\hline & Carmen del Trafún Oeste & TO & 712171 & 5597029 \\
\hline & Río Mañío & RM & 711860 & 5597161 \\
\hline & Champulli & $\mathrm{CH}$ & 708350 & 5596457 \\
\hline & Forestal Maitén & FM & 707795 & 5596191 \\
\hline & El Maitén & MA & 707240 & 5596014 \\
\hline & Represa & RE & 707146 & 5596057 \\
\hline & La Quinta & LQ & 706945 & 559575 \\
\hline & Nuevo Maitén & NM & 706548 & 5596095 \\
\hline \multirow[t]{6}{*}{3} & Cun-Cun Este & $\mathrm{CE}$ & 703554 & 5597017 \\
\hline & Cun-Cun Oeste & $\mathrm{CO}$ & 703088 & 5597452 \\
\hline & Malihue Viejo & MV & 700691 & 5597921 \\
\hline & Santa Olga & $\mathrm{SO}$ & 699317 & 5597654 \\
\hline & Anita María & $\mathrm{AM}$ & 697222 & 5595025 \\
\hline & Chacaipulli & $\mathrm{CP}$ & 695988 & 5593261 \\
\hline \multirow[t]{9}{*}{4} & Balsa Norte & BN & 695694 & 5592383 \\
\hline & Balsa Sur & BS & 694813 & 5591543 \\
\hline & El Mosqueto & EM & 694546 & 5590769 \\
\hline & Cuyincahuin & $\mathrm{CU}$ & 693429 & 5586939 \\
\hline & Camping & $\mathrm{CA}$ & 692278 & 5586120 \\
\hline & San Pedro & SP & 691787 & 5586085 \\
\hline & Quinchilca & QI & 692029 & 5586006 \\
\hline & Junta & $\mathrm{JU}$ & 691621 & 5586199 \\
\hline & Cuyincahuin Nuevo & $\mathrm{CN}$ & 692002 & 5586058 \\
\hline
\end{tabular}


TABLA 2. Resumen de colores y marcas realizadas durante el estudio. Color representa la pintura elastomérica utilizada.

TABLE 2. Summary of colors and marks used during the study. Color represents the ink color used.

\begin{tabular}{ccccc} 
& & \multicolumn{4}{c}{ Marca } \\
\cline { 3 - 5 } & & Oct 05 - Oct 06 & Nov 06 - Feb 07 / Jun 07-Ago 07 & Dic 07- Mar 08 \\
\hline Zona & Color & I & II & III \\
\hline Zona 1 & Naranja & Caudal & Pélvica, Dorsal, Anal & Pélvica, Dorsal, Anal \\
Zona 2 & Verde & Caudal & Pélvica, Dorsal, Anal & Pélvica, Dorsal, Anal \\
Zona 3 & Rosa & Caudal & Pélvica, Dorsal, Anal & Pélvica \\
Zona 4 & Amarillo & Caudal & Pélvica, Dorsal, Anal & Pélvica, Dorsal, Anal \\
\hline
\end{tabular}

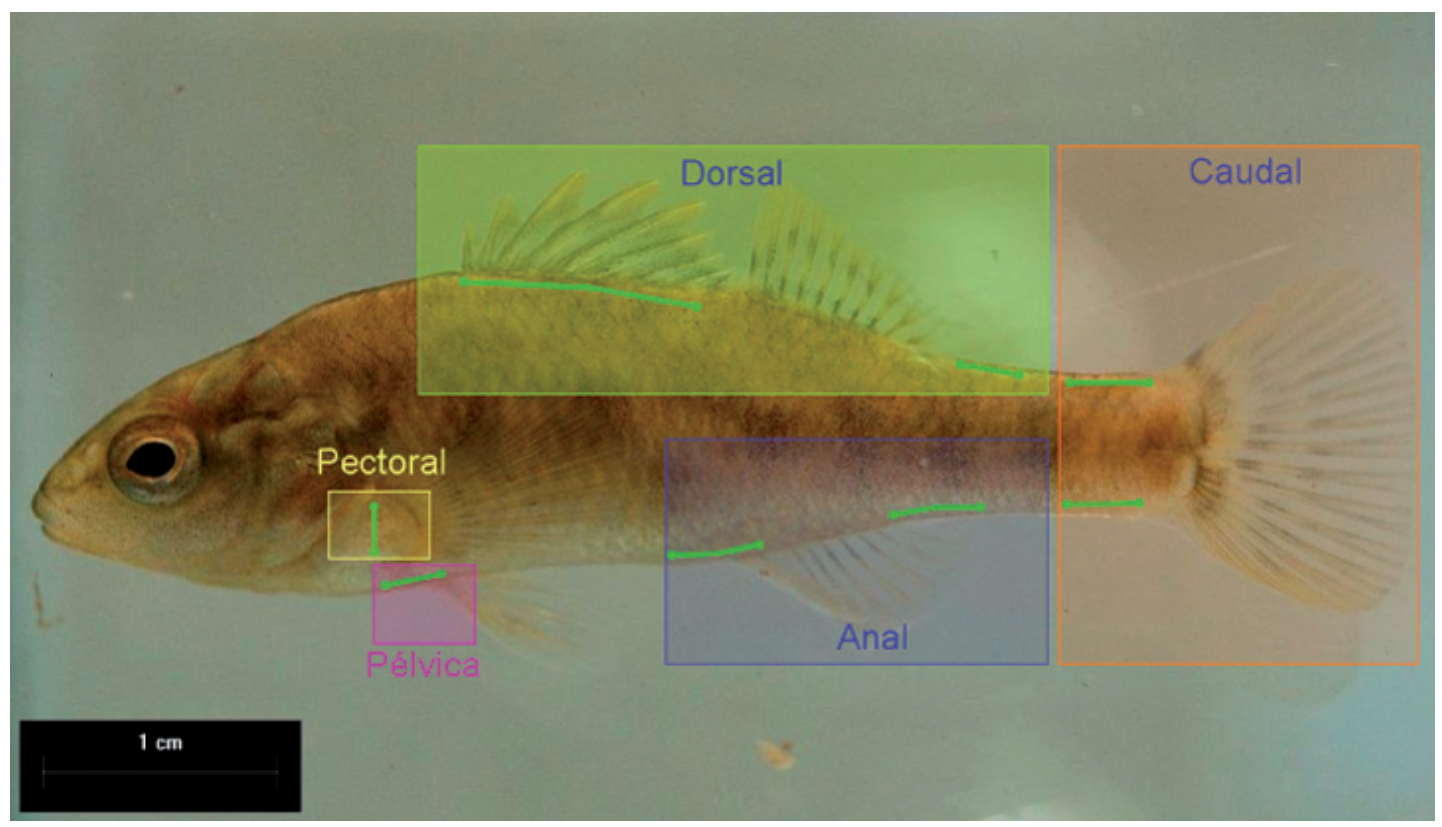

Figura 2. Esquema de las áreas de inyección de elastomer. Se ejemplifica en fotografía de la especie Percilia gillissi.

Figure 2. Body position used for the elastomeric injections. Picture corresponds to the species Percilia gillissi.

\section{RESULTADOS}

MARCAJE - RECAPTURA MEDiAnte Elastomer

Se capturó y devolvió un total de 36.626 individuos a lo largo del río San Pedro, de los cuales 20.190 fueron marcaron con elastomer. Los ejemplares marcados correspondieron a las siguientes especies nativas: A. taeniatus, A. zebra, B. australis, D. camposensis, G. maculatus, G. platei, P.gillissi, y Percichthys trucha (Fig. 3a). Las tallas promedio de los individuos marcados fluctuaron entre $4,0 \mathrm{~cm}$ para $P$. gillissi y $7,2 \mathrm{~cm}$ para A. zebra (Tabla 3 ).

La recaptura total de individuos fue de 588 peces, equivalente al 2,9\% de los marcados. Las especies con mayor número recapturas correspondieron también a las más abundantes, es decir, G. maculatus y P. gillissi. La especie que tuvo mayor tasa de recaptura total (relación entre el número de individuos capturados y recapturados) correspondió a $A$. zebra con un 6,7\% (Fig. 3a). Esta especie sólo fue capturada en las zonas de marcaje 1 (5 individuos) y 2 (10 individuos) y correspondió a la más rara del área de estudio.

La zona de marcaje 1 fue el área con mayor número de individuos marcados, pero con baja tasa de recaptura (Fig. 4a). Los sitios que presentaron un mayor número de recapturas fueron los localizados en las zonas de marcaje 3 y 4. Las especies con mayor número de recaptura en esas zonas fueron G. maculatus (sitios AM, BS, CA), P. gillissi (AM, CA) y G. platei (CO) (Fig. 4b). Esta última especie también tuvo altas recapturas en los sitios PB y LB de las zonas 1 y 2 , que corresponden al área entre el desagüe del lago y el inicio de los rápidos del río (Fig. 4b). Por el contrario, la 
especie $D$. camposensis tuvo muy bajas recapturas en las todas las zonas y sitios (Fig. 4a, 4b). La recaptura total para G. maculatus (4\%) fue variable entre zonas. En la zona 1 se obtuvo la menor tasa de recaptura, con un $1,1 \%$ para esta especie. En tanto, en las zonas 2 y 4, la tasa de recaptura superó el 5\%, y en la zona 3 alcanzó a un 8,9\% (Fig. 4a). La mayor tasa de recaptura para $G$. platei, ocurrió en las zonas
$2(5,3 \%)$ y $3(6,7 \%)$, aún cuando fue en la zona 1 fue donde se marcó la mayor cantidad de ejemplares (2.184), de los cuales se recapturó sólo el 2,3\% (Fig. 4a). Por su parte, se capturaron 7.108 individuos de $P$. gillissi, con una tasa de recaptura total de $2,4 \%$ (171) y con un promedio de $3,4 \%$ para las zonas 2 y 3 (Fig. 4a).
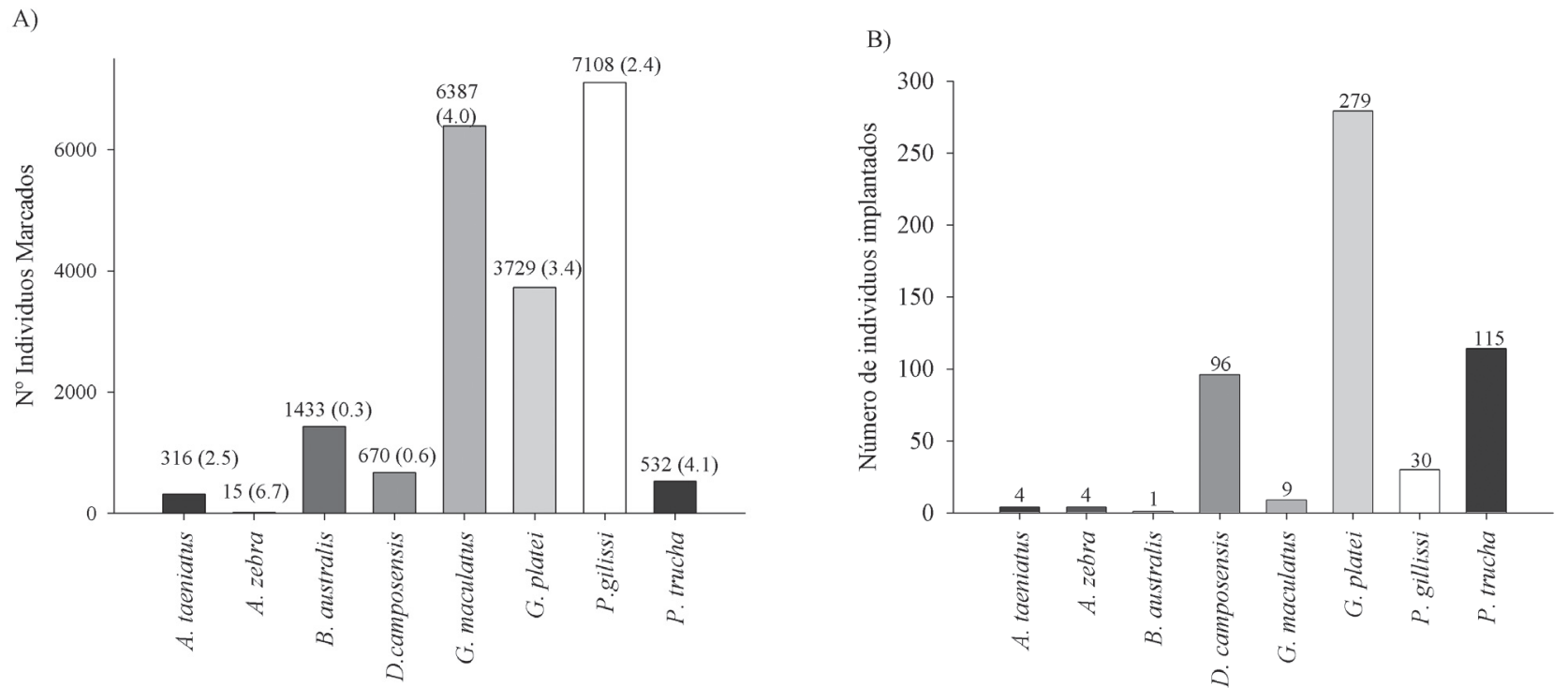

Figura 3. (A) Número de individuos por especie marcados con elastomer y porcentaje de recaptura total entre paréntesis. (B) Número de individuos marcados con Pit-tags.

Figure 3. (A) Number of individuals by species marked with elastomeric inks and recapture percentage in parenthesis. (B) Number of individuals marked with Pit-tags.

TABLA 3. Talla y peso promedio (y error estándar) de los individuos marcados por especie, con cada técnica de marcaje.

TABLE 3. Mean of standard length and weight (and standard error) of the marked individuals with each marking technique.

\begin{tabular}{lcccc}
\hline & \multicolumn{2}{c}{ Talla (cm) } & \multicolumn{2}{c}{ Peso (g) } \\
\hline Elastomer & Promedio & Error estándar & Promedio & Error estándar \\
\hline A. taeniatus & 5,41 & 0,05 & 1,86 & 0,08 \\
A. zebra & 7,19 & 0,27 & 5,32 & 0,67 \\
B. australis & 5,47 & 0,05 & 2,25 & 0,07 \\
D. camposnesis & 5,68 & 0,10 & 5,93 & 0,54 \\
G. maculatus & 5,27 & 0,23 & 1,26 & 0,01 \\
G. platei & 5,57 & 0,03 & 2,43 & 0,07 \\
P. gillissi & 4,03 & 0,01 & 1,53 & 0,01 \\
P.trucha & 5,86 & 0,09 & 5,62 & 0,32 \\
\hline & & & \\
Pit-Tag & & & 6,31 & 0,52 \\
\hline A. taeniatus & 8,02 & 0,36 & 7,08 & 0,20 \\
A. zebra & 7,83 & 0,03 & 648,6 & - \\
B. australis & 33,00 & - & 28,33 & 3,14 \\
D. camposnesis & 10,56 & 0,33 & 5,90 & 0,36 \\
G. maculatus & 7,55 & 1,17 & 12,08 & 0,51 \\
G. platei & 10,56 & 0,15 & 5,97 & 0,22 \\
P. gillissi & 6,98 & 0,15 & 23,64 & 9,57 \\
P. trucha & 8,80 & 0,15 & &
\end{tabular}



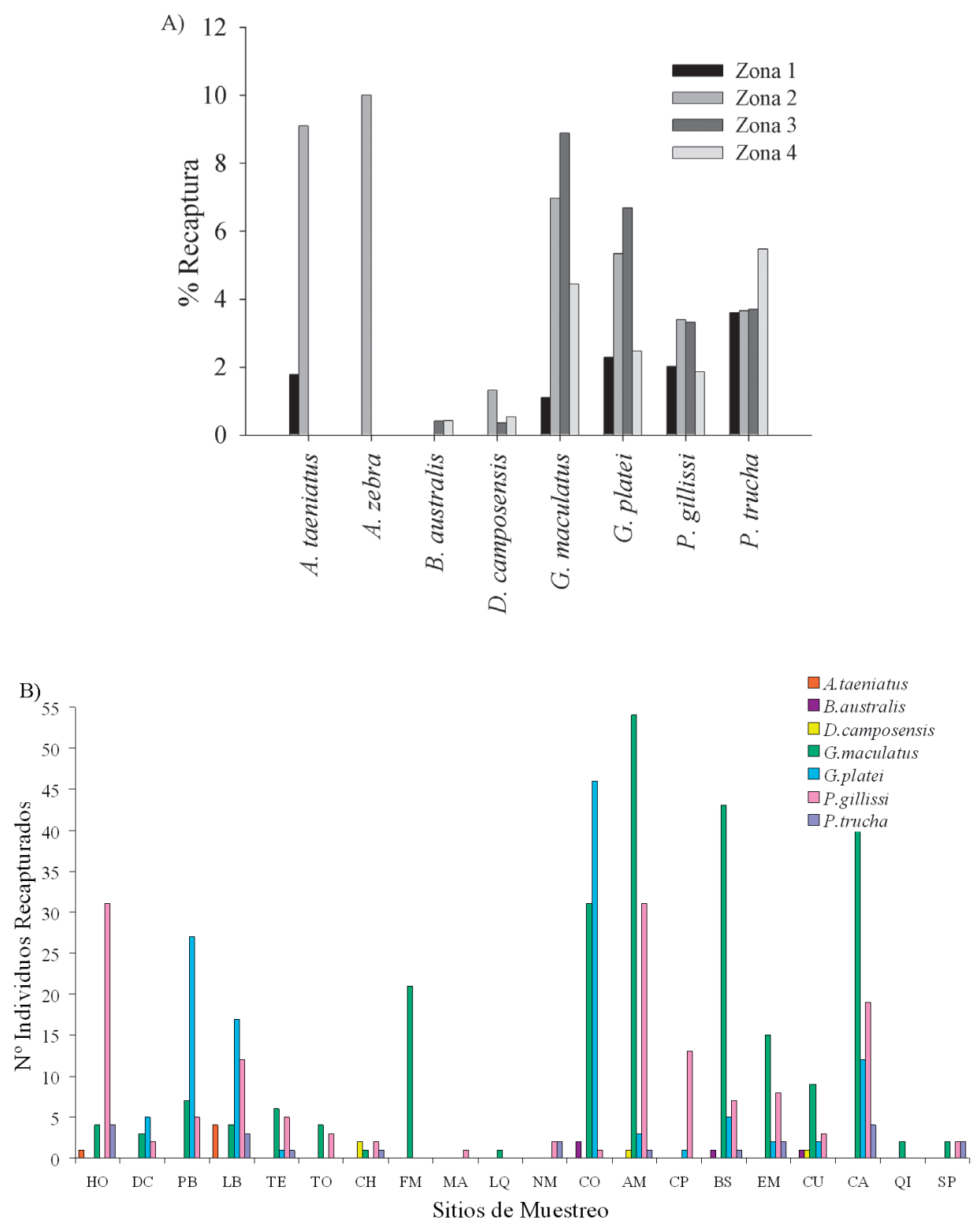

FIgURA 4. (A) Porcentaje y número de individuos recapturados por especie en cada (A) zona de marcaje y (B) sitio de muestreo, ordenados desde el lago Riñihue hacia aguas abajo.

Figure 4. Percentage and number of recaptured individuals by species in each (A) marking zone and (B) sampling sites, going from the Riñihue Lake downstream.

Las especies $B$. australis, P. trucha y D. camposensis, no mostraron evidencia de desplazamientos con esta técnica de marcaje. Para las restantes especies se detectó algún tipo de desplazamiento, los que se describen a continuación. En el caso de $A$. taeniatus se detectaron desplazamientos de dos individuos entre riberas (8,2 cm LS promedio). Estos individuos se desplazaron desde la ribera sur a la norte en sentido de la corriente, a una distancia de 0,2 Km desde el sitio de origen. Además, un individuo se desplazó desde la zona de marcaje 1 hacia la zona 2 (en sentido de la corriente), recorriendo una distancia equivalente a $12,5 \mathrm{Km}$. En $G$. maculatus se registraron 13 individuos (talla promedio de $6,4 \mathrm{~cm} \mathrm{LS}$ ) que presentaron algún tipo de desplazamiento. Los desplazamientos realizados por esta especie en el sentido de la corriente se registraron desde las zonas de marcaje 1, 2 y 3 hacia la zona 4. Es decir, todos entre estos movimientos, un individuo de $6,5 \mathrm{~cm}$ LS pudo haber recorrido una distancia máxima de $37 \mathrm{Km}$ ya que provenía de la Zona 
1 (Fig. 5). Tres individuos se desplazaron desde la zona 2 a las zonas 3 y 4 . Uno de ellos recorrió 19,3 Km, ya que se capturó en el sitio Balsa Sur (BS), el cual corresponde al sitio de la zona 3 ubicado más aguas abajo en el río. Los dos individuos restantes recorrieron una distancia máxima probable de $25 \mathrm{Km}$, desde la zona 2 hasta la 4 (Fig. 5). Para G. platei se detectaron desplazamientos aguas abajo de seis individuos, uno de ellos desde la zona 1 hasta el sitio CA (zona 4), equivalente a una distancia máxima de $38 \mathrm{Km}$ y mínima de $25 \mathrm{Km}$. En menor magnitud se desplazaron cuatro individuos, entre $1,4 \mathrm{Km}$ aguas abajo hasta una distancia máxima probable de $1,9 \mathrm{Km}$. Otro ejemplar se desplazó desde la ribera sur de la zona dos hasta el sitio de LQ de la misma zona, pero ubicado en la ribera norte. Al igual que G. maculatus $y$ G. platei, existieron individuos de P. gillissi que se desplazaron desde la zona 1 a la 3 (26 $\mathrm{Km})$ y desde la zona 2 a la $4(22 \mathrm{Km})$. Para dos individuos existe la posibilidad que se hayan desplazado tanto desde aguas abajo (desde sitio $\mathrm{CH}$ hasta FM) como aguas arriba, desde el sitio LQ a FM, con una distancia de 1,6 y $2 \mathrm{Km}$ respectivamente. Esta posibilidad existe ya que la marca realizada tanto en el sitio de aguas arriba, $\mathrm{CH}$, como en el de aguas abajo (LQ) de FM poseen la misma marca de origen.

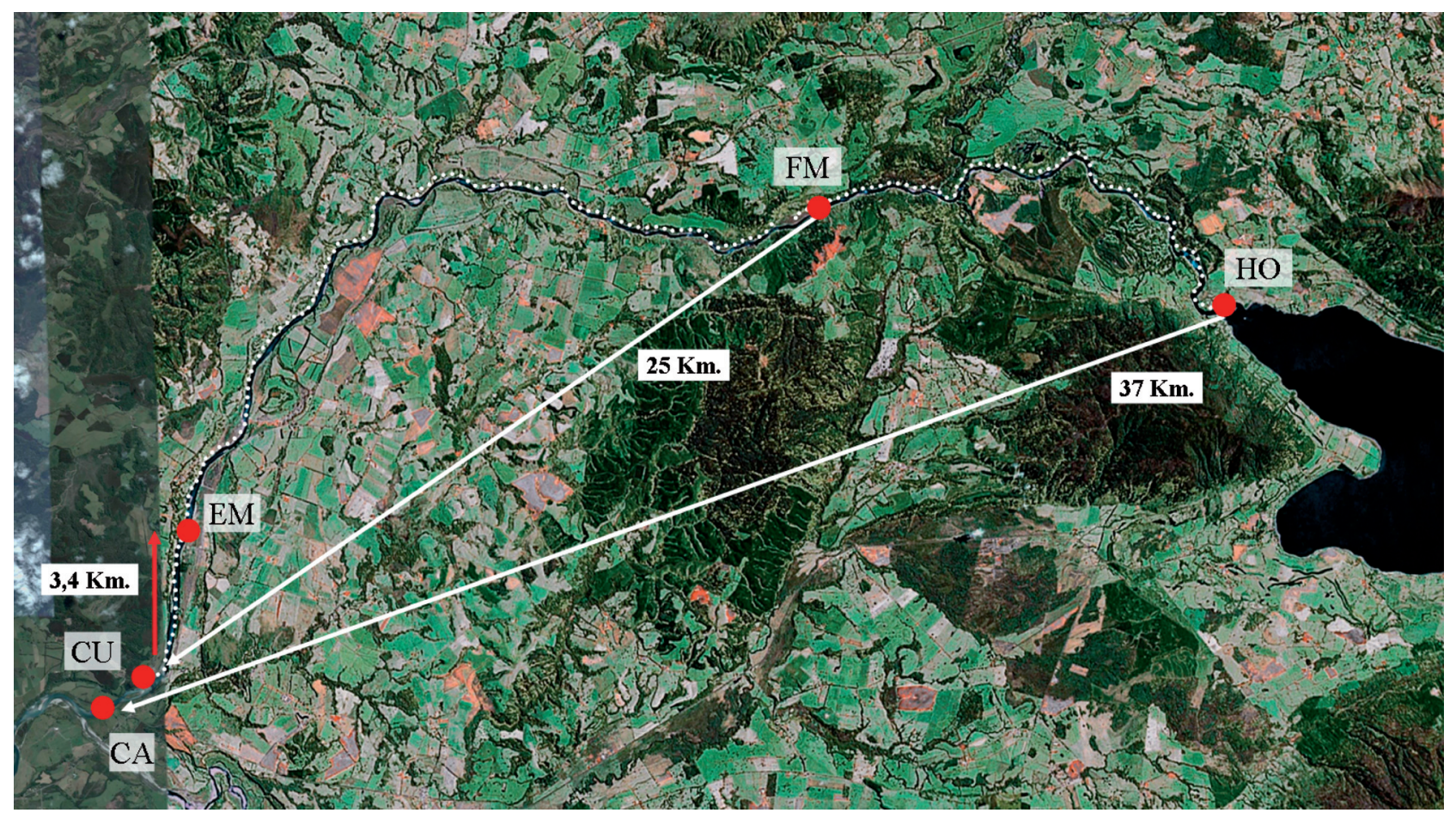

FIGURA 5. Desplazamiento máximo probable realizado por Galaxias maculatus.

Figure 5. Maximum possible movement of Galaxias maculatus.

Las especies que presentaron mayor residencia en una zona a lo largo del río fueron P. gillisi, con un registro mínimo de 26 meses en la zona baja del río, G. maculatus con un periodo mínimo de residencia de 19 meses en zona 4 y G. platei con un periodo mínimo de 15 meses en zona 1 (Fig. 6).

\section{Marcaje Recaptura con Pit-Tags}

Se implantaron 536 peces con Pit-tags, correspondientes principalmente a las especies G. platei (52\%), P. trucha (21\%) y D. camposensis (18\%) (Fig. 3b). Aún cuando $B$. australis alcanza tallas apropiadas para esta técnica, no se utilizó intensivamente, ya que es una especie poco resistente a la manipulación. Las tallas de los individuos marcados con
Pit-tags fluctuaron entre $6,9 \mathrm{~cm}$ para $P$. gillissi y $33 \mathrm{~cm}$ en $B$. australis (Tabla 3).

La tasa de recaptura total para Pit-tags fue baja e igual a $1,49 \%$. Los individuos recapturados correspondieron a tres ejemplares de G. platei y cuatro de P. trucha. Los individuos de $G$. platei fueron recapturados en el mismo sitio de origen (sitio $\mathrm{CO}$ en Zona 3), correspondiente a un hábitat de poza temporal, la cual en época de bajo caudal permanece desconectada del curso principal del río. El crecimiento, y el cambio en biomasa de estos ejemplares recapturados, fue diferente en el periodo marzo-mayo que en el periodo mayo-diciembre (Tabla 3, Fig. 7a). 
De los cuatro individuos recapturados de $P$. trucha $(3,6 \%)$, uno provenía de la Zona 1 de marcaje y 3 de la Zona 2 . Todos los ejemplares fueron marcados y recapturados en la mismo sitio, en hábitats de pozas en un período no superior a 47 días (Tabla 3, Fig. 7b). No se obtuvo recapturas de ejemplares marcados en lapsos de tiempo mayores. El incremento en talla fue muy similar en los cuatro peces recapturados. Sin embargo, un ejemplar presentó un incremento diario de peso superior, probablemente debido a la ingestión reciente de alimento.

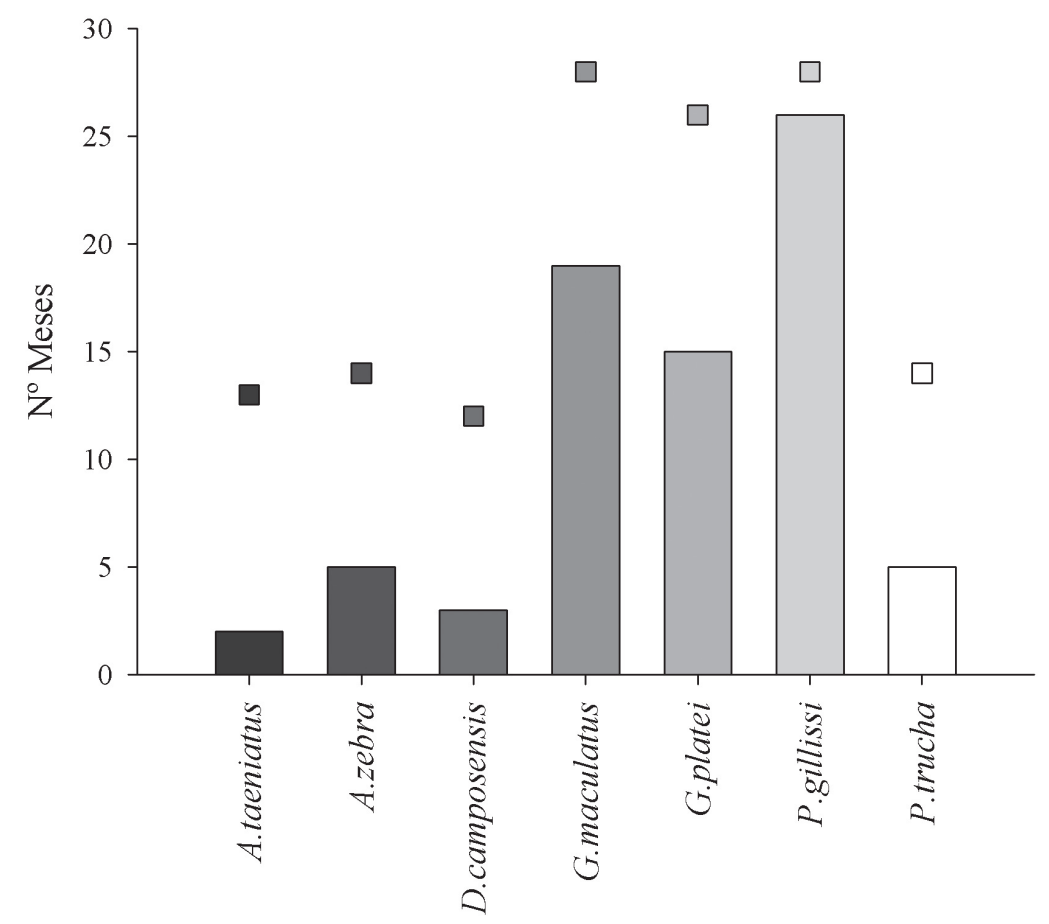

FIgURA 6. Periodos mínimos (barras) y máximo (cuadros) de residencia por especie durante el estudio.

FIGURE 6. Minimum (bars) and maximum (squares) times residency by species during the study.

A)

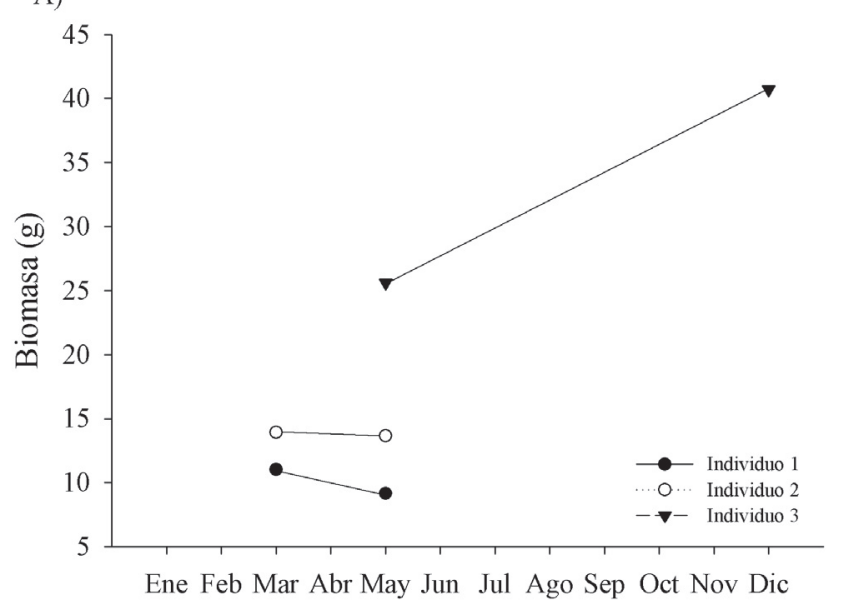

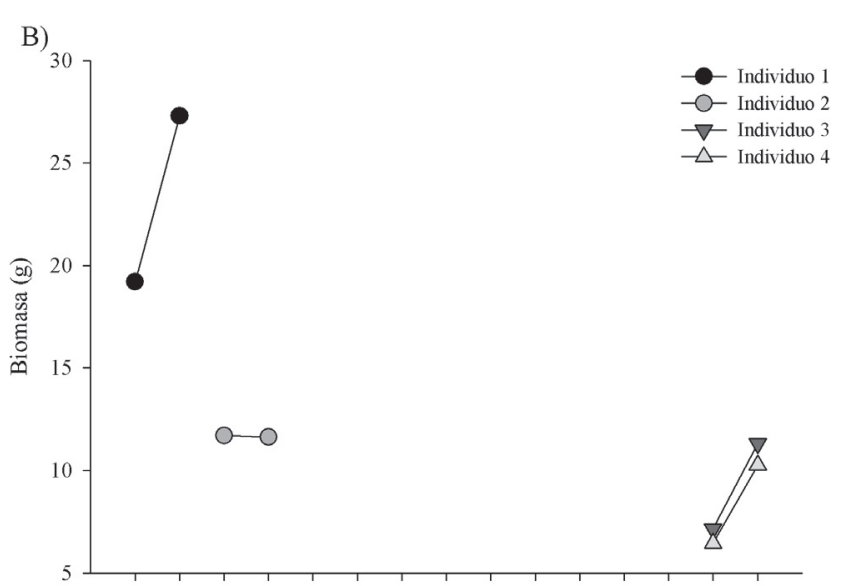

Ene Feb Mar Abr May Jun Jul Ago Sep Oct Nov Dic Ene

FIGURA 7. Cambios en biomasa registrados por ejemplares marcados con Pit-tags de (A) Galaxias platei y (B) Percichthys trucha.

FIGURE 7. Biomass changes of individuals of (A) Galaxias platei and (B) Percichthys trucha marked with Pit-tags. 


\section{DISCUSION}

Si bien, las técnicas de marcaje-recaptura utilizadas entregan registros relevantes, ellas deben ser complementadas con otras técnicas indicativas de patrones de desplazamiento. En este sentido, aquí analizamos nuestros resultados a la luz de las características genético-poblacionales y la distribución de cada especie en la cuenca (Cifuentes et al. 2012; Victoriano et al. 2012; Colin et al. 2012). De acuerdo a nuestros resultados, la mayoría de las especies nativas estudiadas presentan movimientos que pueden ser extensos, pero en el sentido de la corriente o entre riberas. Resulta destacable que peces de pequeño tamaño $(<10 \mathrm{~cm}$ LS) puedan desplazarse hasta aproximadamente $40 \mathrm{~km}$ en el sentido de la corriente, sobreviviendo en buenas condiciones en nuevos hábitats ubicados aguas abajo. Por ello, estos movimientos son de gran relevancia para la colonización continua de nuevos hábitats (Hancock et al. 1999; Smithson et al. 1999). De igual manera, el flujo unidireccional de individuos no sólo tiene sentido ecológico, sino que también implica un importante aporte de genes desde poblaciones fuentes, ubicadas en las zonas altas de la cuenca, hacia las zonas bajas (Vrijenhoek 1998). Todos estos procesos son fundamentales para la mantención de las dinámicas metapoblacionales en sistemas complejos como los ríos (Hanski \& Gaggiotti 2004). Más aún, es posible que estos movimientos sean voluntarios y no pasivos como se ha asumido extensamente en la literatura (Rice et al. 2010).

Los resultados obtenidos muestran dinámicas poblacionales distintas para las diferentes especies. Así por ejemplo, el bajo número de individuos capturados y marcados de las especies del género Aplochiton, sumado a su alta tasa de recaptura, la mayoría obtenidas en la misma zona, son indicativos de tamaños poblacionales pequeños y baja movilidad. Sin embargo, los individuos marcados correspondieron a juveniles, por lo que es posible que una vez alcanzada la madurez sexual éstos se desplacen hacia los lagos ubicados aguas arriba. De hecho, el hábitat descrito para adultos corresponde a lagos (Campos 1969; Habit et al. 2010), y la gran proporción de individuos que se capturan en el río San Pedro son juveniles (Cifuentes et al. 2012). Por ello, postulamos que los ejemplares maduros de estas especies son altamente móviles y se desplazan posiblemente aguas abajo desde los lagos hacia el río y viceversa.

Por su parte, la especie G. platei mostró únicamente movimientos a favor de la corriente. El hallazgo de un grupo de individuos que se encontraron por aproximadamente un año en una poza protegida en el río, sugiere que los movimientos aguas abajo son involuntarios y debidos al arrastre mecánico. Por otra parte, consistentemente con la menor tasa de recaptura en la zona 1, la mayor abundancia de esta especie en el área ocurre en las cercanías del lago Riñihue
(Colin et al. 2012). Tal población funcionaría como fuente de aquellas ubicadas río abajo. De hecho, G. platei, a diferencia de G. maculatus, no presenta estructuración genética entre la población del lago Riñihue y del río San Pedro (Victoriano et al.2012), por lo que estos desplazamientos hacia aguas abajo, ayudarían a mantener el flujo génico a lo largo del río.

Los resultados obtenidos para G. maculatus sugieren un comportamiento diferencial de la especie a lo largo del área de estudio, lo cual se evidencia también en sus características genéticas (Victoriano et al. 2012). La información combinada sugiere que la población de G. maculatus que habita en el lago Riñihue es de gran tamaño y baja movilidad o residente como fue descrito por Campos (1974). En tanto, el menor número de individuos capturados y marcados en la zona 3 , pero una mayor tasa de recaptura en ella, sugieren una población de menor tamaño y menos móvil, probablemente residente del río. Estos datos también son consistentes con la estructura genética de las poblaciones en la cuenca, que sugieren poblaciones relativamente aisladas entre el lago, río y zona estuarina (Victoriano et al. 2012). La conexión entre estas poblaciones estaría explicada principalmente a través de los movimientos en el sentido de la corriente, y en menor grado por movimientos en contra de la corriente de menor envergadura.

Las especies $D$. camposensis y $B$. australis presentaron las tasas de recapturas más bajas respecto de las demás especies marcadas. Al complementar estos resultados para $D$. camposensis con los obtenidos por Oyanedel (com. pers.), quienes analizaron desplazamientos mediante telemetría, la baja abundancia de la especie reportada por Habit et al. (2009) en el mismo sistema y su alto flujo génico a lo largo del río (Victoriano et al. 2012), postulamos que esta es una especie de muy alta movilidad. Por ende, es también una de las más altamente amenazadas por la presencia de barreras en el río, tales como presas de centrales hidroeléctricas. Por su parte, la baja tasa de recaptura de B. australis es indicativa de una gran población y de alta movilidad a lo largo del río, lo cual es consistente con su abundancia (Colin et al. 2012) y falta de estructura genética en el área de estudio (Victoriano et al. 2012).

Al igual que para las especies anteriores, la baja tasa de recaptura de $P$. gillissi es indicativa de su gran tamaño poblacional (Colin et al. 2012). Ello, sumado a la ausencia de individuos recapturados en zonas aguas arriba o abajo de su zona de marcaje indican una escasa capacidad de desplazamiento. Sin embargo, los análisis genéticos demuestran un alto flujo génico a lo largo del río San Pedro para esta especie (Victoriano et al. 2012). Ello se explicaría, tanto por su gran tamaño poblacional como por el traslape de eventos de reproducción en pequeños tramos a lo largo del río. A diferencia de ésta, $P$. trucha, obtuvo un bajo número 
de individuos marcados y alta proporción de recapturas lo que indica que la población de $P$. trucha es pequeña en el río $\mathrm{y}$, probablemente de baja movilidad. Esto es consistente con los patrones genéticos (Victoriano et al. 2012), los cuales indican una estructuración de las poblaciones entre la zona del lago y río San Pedro.

En general, la baja tasa de recaptura de individuos marcados con Pit-tags puede estar relacionada con la pérdida o eliminación del chip. Así por ejemplo, Baras \& Westerloppe (1999), comprobaron en Heterobancus longifilis (Siluridae), la expulsión del chip por el intestino. Esto ocurre primero con un desarrollo de una cápsula alrededor del Pit-tag, la cual se adhiere al intestino pasando al interior de él, el cual es expulsado posteriormente por movimientos peristálticos. Tal situación fue observada en individuos de G. platei, quienes presentaban el Pit-tag en el intestino, y cerca de ser expulsado completamente.

Finalmente, concluimos que los peces presentes en el río San Pedro, a pesar de su pequeño tamaño corporal, realizan desplazamientos de gran magnitud, los cuales deben ser considerados para comprender su ecología y requerimientos de hábitat en sistemas fluviales altamente intervenidos como los ríos de la zona centro sur de Chile.

\section{AGRADECIMIENTOS}

Agradecemos a COLBUN S.A. por financiar este estudio y al proyecto DIUC Semilla Patagonia 210.310.057-1SP por el financiamiento de la publicación. A los propietarios de los predios aledaños al río por permitirnos ingresar a ellos, y muy especialmente al Hotel Riñimapu por las facilidades brindadas para llevar a cabo esta investigación. Finalmente se agradece al proyecto Fondecyt 1110441 que permitió la finalización de este manuscrito.

\section{BIBLIOGRAFIA}

Albanese, B., Angermeier, P. \& Dorai-Raj, S. 2004. Ecological correlates of fish movement in a network of Virginia streams. Canadian Journal Fish Aquatic Science 61:857869.

Allen, M.B., Connolly, P., Mesa, M., Charrier, J. \& Dixon, C. 2010. Distribution and movement of bull trout in the upper Jarbidge River watershed, Nevada: U.S. Geological Survey Open-File Report 2010-1033. 80 pp.

Baras, E. \& Westerloppe, L. 1999. Transintestinal expulsion of surgically implanted tags in African catfishes of different size and age. Transactions of the American Fisheries Society 128: 737 -746.

Beamish, F.W.H. 1978. Swimming capacity. Fish Physiology 7:101-187

Campos, H. 1969. Reproducción del Aplochiton taeniatus. Boletín del Museo Nacional de Historia Natural 29: 208-222.

Campos, H.1974. Populations Studies of Galaxias maculatus (Jenyns) (Osteichthys:Galaxiidae) in Chile with Reference to the number of Vertebrae. Studies on the Neotropical Fauna and Environment 9:55-76.

Cifuentes, R., Gonzalez, J., Montoya, G., Jara, A., Ortíz, N. P. PIEDRA \& HABIT, E. 2012. Relación Longitud-peso y factor de condición de los peces nativos del río San Pedro cuenca del río Valdivia, Chile). Gayana 76 (Número Especial): 101-110.

Colin, N., Piedra, P. \& Habit. E. 2012.Variaciones espaciales y temporales de las comunidades ribereñas de peces en un sistema fluvial no intervenido: Río San Pedro, Cuenca del Valdivia (Chile). Gayana 76 (Número Especial): 24-35.

EILER, J. 2000. Fish movements - the missing piece. In Fish Movement and Migration. Australian Society for Fish Biology Workshop Proceedings, Bendigo, Victoria, September 1999, Australian Society for Fish Biology. D. A. Hancock, D. C. Smith and J. D. Koehn, Sydney 85-92 pp.

Gowan, C., Young, M., Fausch, F. \& Riley, S. 1994. Restricted movement in resident stream salmonids: a paradigm lost? Canadian Journal of Fisheries and Aquatic Sciences 47: 1724-1737.

Habit E., Jara, A., Colin, N., Oyanedel, A., Victoriano, P., Gonzalez, J. \& Solis-Lufí, K. 2009. Threatened fishes of the world: Diplomystes camposensis Arratia,1987 (Diplomystidae) Environmental Biology of Fishes 84: 393-394.

Habit, E., Piedra, P., Ruzzante, D.E., Walde, S., Belk, M., Cussac, V., Gonzalez, J. \& Colin, N. 2010. Changes in the distribution of native fishes in response to introduced species and other anthropogenic effects. Global Ecology and Biogeography 19: 697-710.

Hancock, D.A., D. Smith \& J.D. Koenn. 1999. Fish movement and Migration. Australian Society for Fish Biology Workshop Proceedings, Bendigo, Victoria, 28-29 September, 1999. Australian Society for Fish Biology, Sydney.

Hanski, I. \& GagGiotTi, O. 2004. Ecology, genetics \& evolution of metapopulations. (eds. Hanski,I. \& Gaggiotti,O.), Elsiever Academic Press, Amsterdam, 696 pp

Lauder G.V. 2005. Locomotion In The Physiology of Fishes. (Eds. Evans, D.H. \& Clairbone, J.B.), CRC Press, Boca Raton 3-46 pp.

Lonzarich, D., Warren, M. \& LonZarich, M. 1998. Effects of habitat isolation on the recovery of fish assemblages in experimentally defaunated stream pools in Arkansas. Canadian Journal of Fisheries and Aquatic Sciences 55:2141-2149.

LuCAS, M. \& Baras, E. 2000. Methods for studying the spatial behaviour of freshwater fishes in the natural environment. Fish and Fisheries 1: 283-316.

McDowall, RM. 2002. Accumulating evidence for a dispersal biogeography of southern cool temperate freshwater fishes. Journal of Biogeography 29: 207-220.

Niemeyer, H. \& Cereceda, P. 1984. Hidrografía. Geografía de Chile. Tomo IV. Instituto Geográfico Militar, Chile

Rice, S., Lancaster, J. \& Klemp, P. 2009. Experimentation at the interface of fluvial geomorphology, stream ecology and hydraulic engineering and the development of an effective, 
interdisciplinary river science. Earth Surface Processes Landforms 35:64-77.

RodrigueZ, M. 2002. Restricted movement in stream fish: the paradigm is incomplete, not lost. Ecology 83: 1-13.

SCHLOSSER, I.1991. Stream fish ecology: a landscape perspective. BioScience 41: 704-712.

Schlosser, J. \& Angermeier P. 1995. Spatial variation in demographic processes in lotic fishes: Conceptual models, empirical evidence, and implications for conservation. American Fisheries Society Symposium 17: 360-370.

Smithson, E. \& Johnston, C. 1999. Movement patterns of stream fishes in a Ouachita Highlands stream: an examination of the restricted movement paradigm. Transactions of the
American Fisheries Society 128:847-853.

TAYlor, C.1997. Fish species richness and incidence patterns in isolated and connected stream pools: effects of pool volume and spatial position. Oecologia 110:560-566.

Victoriano, P., Vera, I., Olmos, V., Dib, M., Insunza B., MuñozRamírez, C., Montoya, R., Jara, A. \& E. Наbit. 2012. Patrones de diversidad genética de los peces nativos del río San Pedro. Gayana 76 (Número Especial): 71-85.

Vila, I., Fuentes, L. \& Contreras, M. 1999. Peces límnicos de Chile. Boletín del Museo Nacional de Historia Natural, Chile 48: 61-75.

VRIJENHOEK, R.C. 1998. Conservation genetics of freshwater fish. Journal of Fish Biology 53: 394-412.

Recibido: 02.01 .12

Aceptado: 27.02.12 